\title{
Adaptive strategies for solving parameterized systems using homotopy continuation
}

\author{
Jonathan D. Hauenstein* Margaret H. Regan ${ }^{\dagger}$
}

October 1, 2018

\begin{abstract}
Three aspects of applying homotopy continuation, which is commonly used to solve parameterized systems of polynomial equations, are investigated. First, for parameterized systems which are homogeneous, we investigate options for performing computations on an adaptively chosen affine coordinate patch. Second, for parameterized systems which are overdetermined, we investigate options for adaptively selecting a well-constrained subsystem to restore numerical stability. Finally, since one is typically interested in only computing real solutions for parameterized problems which arise from applications, we investigate a scheme for heuristically identifying solution paths which appear to be ending at nonreal solutions and truncating them. We demonstrate these three aspects on two problems arising in computer vision.

Keywords. Numerical algebraic geometry, homotopy continuation, parameter homotopy, overdetermined system, algebraic vision
\end{abstract}

AMS Subject Classification. 65H10, 68W30, 14Q99

\section{Introduction}

Parameterized systems of polynomial equations arise in many applications including computer vision [15, 19,26], chemistry [1,22], and kinematics [12,31]. For a general setup, we assume that $F(x ; p)$ is a system which is polynomial in the variables $x \in \mathbb{C}^{N}$ and analytic in the parameters $p \in \mathbb{C}^{P}$. Typically, one is interested in efficiently computing the solutions for many instances of the parameters. For example, in computer vision, algorithms are used to solve the same system at many parameter values in order to employ the RANSAC algorithm [9, 17].

An approach to repeatedly solve many instances of a parameterized system is to utilize a socalled Gröbner trace approach, e.g., see [17]. That is, one first performs algebraic manipulation of the equations at a randomly selected parameter value to discover how to reduce the corresponding system to a Gröbner basis from which the solutions can be efficiently extracted. The identification of the algebraic manipulation steps form the ab initio phase and is completed "offline." The "online" phase is to repeat these same manipulations for each given parameter instance. This approach can

${ }^{*}$ Department of Applied and Computational Mathematics and Statistics, University of Notre Dame, Notre Dame, IN 46556 (hauenstein@nd.edu, www.nd.edu/ jhauenst). This author was supported in part by Army YIP W911NF15-1-0219, Sloan Research Fellowship BR2014-110 TR14, NSF grant ACI-1460032, and ONR N00014-16-1-2722.

${ }^{\dagger}$ Department of Applied and Computational Mathematics and Statistics, University of Notre Dame, Notre Dame, IN 46556 (mregan9@nd.edu, www.nd.edu/ mregan9). This author was supported in part by Schmitt Leadership Fellowship in Science and Engineering and NSF grant ACI-1440607. 


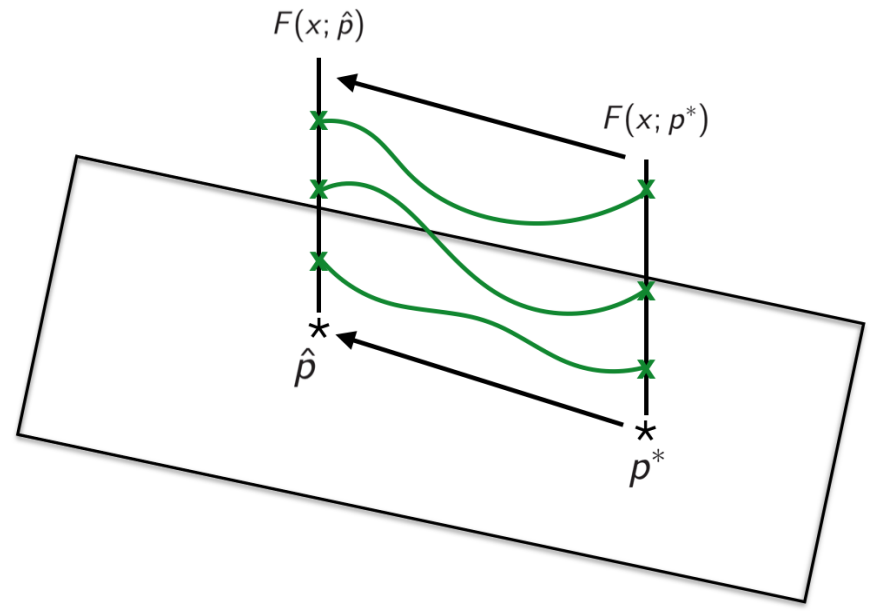

Figure 1: Parameter homotopy phase starting with the solutions of $F\left(x ; p^{*}\right)=0$ and ending with the solutions of $F(x ; \hat{p})=0$.

lead to efficient solvers in computer vision with many potential problems including the need for specialized software, a propagation of errors, instability, and expensive computations 17 .

Rather than employ algebraic manipulation, we consider using homotopy continuation in the form of a parameter homotopy 23] (see also [4, Chap. 6]). To employ a parameter homotopy, the "offline" ab initio phase computes all solutions at a randomly selected parameter value, say $p^{*}$. The "online" parameter homotopy phase tracks the solution paths using homotopy continuation as $p^{*}$ is deformed to the given parameter instance, say $\hat{p}$, which is shown schematically in Figure 1 .

Although parameter homotopies have been used to solve many instances of a parameterized system [2,3], there are three aspects of this computation that warrant further consideration. First, it is common for the parameterized system $F(x ; p)$ to be homogeneous with respect to the variables $x$ so that one treats the solutions as points in the projective space $\mathbb{P}^{N-1}=\mathbb{P}\left(\mathbb{C}^{N}\right)$. For example, $3 \times 3$ essential matrices in $3 \mathrm{D}$ image reconstruction are naturally considered as points in $\mathbb{P}^{8}=\mathbb{P}\left(\mathbb{C}^{3 \times 3}\right)=\mathbb{P}\left(\mathbb{C}^{9}\right)[6$. For problems which are naturally formulated affinely, projective space is used to improve the solving process, particularly for solutions with large norm as well as handling nongeneric parameter values which have solutions at infinity 21. Computationally, one natural approach for handling projective space is to utilize an affine coordinate patch. We will consider three strategies for selecting an affine patch: a fixed coordinate patch which is used throughout the computation 21] (Section 3.1), an adaptive orthogonal patch 25] (Section 3.2), and an adaptive coordinate-wise patch proposed in Section 3.3 .

Second, it is common for parameterized problems to be overdetermined. For example, the set of essential matrices in computer vision consists of $3 \times 3$ matrices of rank 2 where the two nonzero singular values are equal. Since scaling is irrelevant, as mentioned above, this set is naturally defined on $\mathbb{P}^{8}$ by the vanishing of the determinant and the 9 cubic Demazure polynomials [6], namely

$$
2 E E^{T} E-\operatorname{trace}\left(E E^{T}\right) E=0 .
$$

This system of 10 polynomials is overdetermined since it defines an irreducible set of codimension 3 . Due to the numerical instability of solving overdetermined systems, we explore three techniques for reducing down to solving well-constrained systems: a fixed global randomization 27] (Section 4.1), 
an adaptive pseudoinverse randomization proposed in Section 4.2, and an adaptive leverage score randomization proposed in Section 4.3 .

Third, when solving parameterized problems arising from applications, typically only the real solutions are of interest. That is, one need not compute the nonreal endpoints of solution paths defined by a homotopy. We propose a heuristic strategy in Section 6 for identifying and truncating paths which appear to be ending at nonreal solutions thereby saving computational time.

The remainder of the paper is as follows. Section 2 provides a short introduction to parameter homotopies and path tracking with more details provided in [4,28. Section 3 compares the three strategies for affine patches while Section 4 compares the three strategies for randomizing down to a well-constrained subsystem. Section 5 presents pseudocode for the path tracking methods with Section 6 presenting our heuristic truncation scheme for nonreal solutions. We compare all of the approaches on two applications in computer vision in Section 7. The paper concludes in Section 8 .

\section{Parameter homotopies and path tracking}

Throughout, we assume that the parameterized system $F(x ; p)$ is polynomial in the variables $x \in \mathbb{C}^{N}$ and analytic in the parameters $p \in \mathbb{C}^{P}$. This setup ensures that the number of nonsingular isolated solutions of $F=0$ has a generic behavior with respect to the parameter space $\mathbb{C}^{P}$, e.g., 28 , Thm. 7.1.5]. This enables path tracking on the parameter space via a parameter homotopy 23 ] described below. We note that one could also consider positive-dimensional components using linear slicing and singular isolated solutions using deflation techniques, e.g., [11, 20], to reduce to the nonsingular isolated case.

For generic $p^{*} \in \mathbb{C}^{P}$, the ab initio phase of parameter homotopy continuation is to compute the isolated nonsingular solutions of $F\left(x ; p^{*}\right)=0$. This can be accomplished, for example, using standard homotopy continuation [4,28] which is a computation that is performed once "offline."

Given $\hat{p} \in \mathbb{C}^{P}$, the "online" part is called the parameter homotopy phase which computes the isolated nonsingular solutions of $F(x ; \hat{p})=0$ using the parameter homotopy

$$
H(x, t)=F\left(x ; t p^{*}+(1-t) \hat{p}\right) .
$$

In particular, for each nonsingular solution $x^{*}$ of $F\left(x ; p^{*}\right)=0$, one considers the solution path $x(t)$ defined by $x(1)=x^{*}$ and $H(x(t), t) \equiv 0$. Genericity of $p^{*}$ ensures that each solution path $x(t)$ is smooth for $t \in(0,1]$ and satisfies the Davidenko differential equation

$$
J_{x} H(x, t) \cdot \dot{x}(t)=-J_{t} H(x, t)
$$

where $J_{x} H(x, t)$ and $J_{t} H(x, t)$ are the Jacobian matrix with respect to $x$ and Jacobian vector with respect to $t$, respectively. Hence, one can employ a predictor-corrector tracking strategy starting

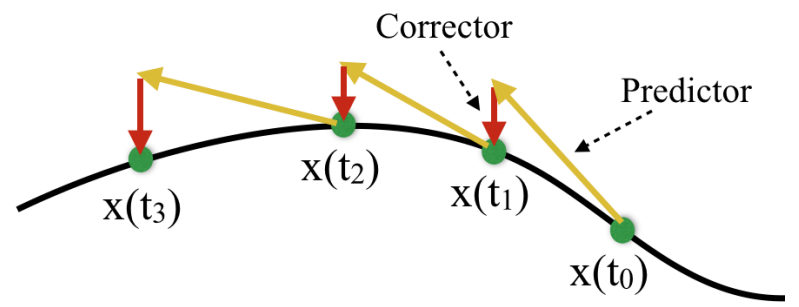

Figure 2: A schematic view of predictor-corrector tracking along a solution path. 
with the initial value $x(1)=x^{*}$ to compute $x(0)$. A schematic view of predictor-corrector tracking is provided in Figure 2 with more details provided in [4, 28]. In particular, the predictor follows from the differential equation (3) while the corrector uses the fact that $H(x(t), t) \equiv 0$. Since, for $t \in(0,1], x(t)$ is a nonsingular isolated solution of $H(\bullet, t)=0$, Newton's method is locally quadratically convergent. Hence, our computations will utilize classical $4^{\text {th }}$ order Runge-Kutta prediction method with the corrector being several iterations Newton's method.

\section{Affine patches}

The projective space $\mathbb{P}^{N}$ is the set of lines in $\mathbb{C}^{N+1}$ passing through the origin. In particular, there is a choice to be made for performing computations on $\mathbb{P}^{N}$ due to selecting a representation of each point in $\mathbb{P}^{N}$. One standard approach is to utilize an affine coordinate patch where a Zariski open dense subset of $\mathbb{P}^{N}$ is represented by a hyperplane in $\mathbb{C}^{N+1}$ as illustrated in the following.

Example 3.1 Consider intersecting the twisted cubic curve $C \subset \mathbb{P}^{3}$ with the hyperplane defined by $x_{0}+x_{1}+x_{2}+x_{3}=0$, namely computing the three solutions on $\mathbb{P}^{3}$ of the polynomial system

$$
f(x)=\left[\begin{array}{c}
x_{0} x_{2}-x_{1}^{2} \\
x_{1} x_{2}-x_{0} x_{3} \\
x_{1} x_{3}-x_{2}^{2} \\
x_{0}+x_{1}+x_{2}+x_{3}
\end{array}\right]=0
$$

which are

$$
[1,-1,1,-1],[1, i,-1,-i],[1,-i,-1, i] \in \mathbb{P}^{3}
$$

where $i=\sqrt{-1}$. In (5), each point in $\mathbb{P}^{3}$ is represented by a unique vector in $\mathbb{C}^{4}$ using the affine coordinate patch defined by $x_{0}=1$, i.e., represented uniquely in the form $\left[1, x_{1}, x_{2}, x_{3}\right]$. The set of points in $\left[x_{0}, x_{1}, x_{2}, x_{3}\right] \in \mathbb{P}^{3}$ which cannot be represented in this way is the hyperplane $x_{0}=0$.

The key to selecting an affine coordinate patch is to make sure that every projective point of interest, e.g., every point along every homotopy solution path, has a representation in that affine patch. For example, the first point in (5) cannot be represented in the affine coordinate patch defined by $x_{0}+x_{1}=1$.

The following describes three strategies for selecting an affine patch. The first uses a fixed general affine patch [21] while the second and third utilize a locally adapted orthogonal [25] and coordinate-wise patching strategy, respectively.

\subsection{Fixed general affine patch}

The approach presented in 21] uses a general affine coordinate patch. That is, for a general $v \in \mathbb{C}^{N+1}$, one performs all computations on the fixed affine coordinate patch defined by

$$
v \cdot x=v^{H} x=1
$$

where $v^{H}$ is the Hermitian (conjugate) transpose of $v$. See Figure 3(a) for a schematic view.

The advantage of using a fixed affine coordinate patch is that it is chosen at the beginning and is fixed throughout the computations. Thus, one could perform computations implicitly on the patch which removes one of the variables, e.g., remove the variable $x_{0}$ via

$$
x_{0}=\frac{1}{\operatorname{conj}\left(v_{0}\right)}\left(1-\operatorname{conj}\left(v_{1}\right) x_{1}-\cdots-\operatorname{conj}\left(v_{N}\right) x_{N}\right) .
$$




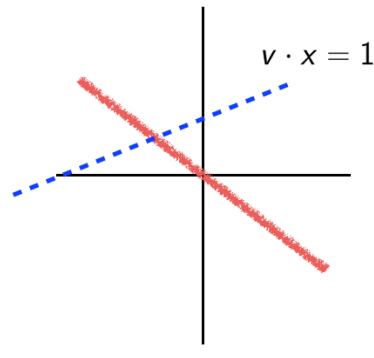

(a)

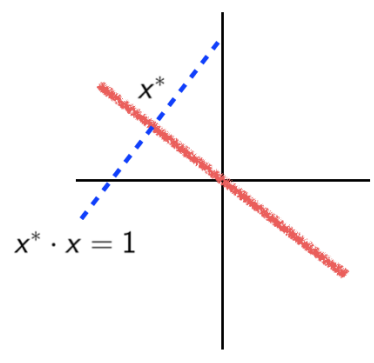

(b)

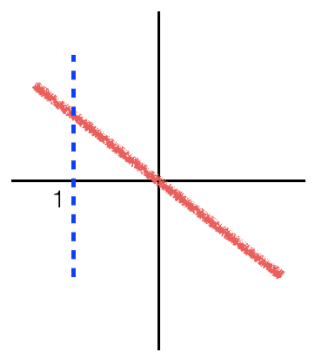

(c)

Figure 3: Schematic drawing of (a) fixed affine patch, (b) orthogonal affine patch, and (c) coordinate-wise affine patch.

The disadvantage of using a fixed patch is that ill-conditioning could artificially be introduced as shown in the following.

Example 3.2 With $f(x)$ as in (4), consider the system of equations

$$
g(x)=\left[\begin{array}{c}
f(x) \\
v \cdot x-1
\end{array}\right]=0 .
$$

Table 1 shows the condition number with respect to the 2-norm of the Jacobian matrix of $g$ using various vectors $v$ at the solution corresponding to $[1,-1,1,-1]$ in the respective affine patches.

\begin{tabular}{c|c|c|c}
$v$ & $(1,0,0,0)$ & $(0.8695,0.4670,-0.0231,0.1592)$ & $(0.1947,0.3999,-0.5268,-0.7243)$ \\
\hline $\mathrm{CN}$ & 10.2 & 158.2 & $113,574.2$
\end{tabular}

Table 1: Condition number of the Jacobian matrix of $g$ with respect to different coordinate patches

One can attempt to limit this artificial ill-conditioning by using a locally selected affine coordinate patch, i.e., one which is adapted to the current point to be represented. The next two subsections consider two methods for selecting local patches.

\subsection{Orthogonal affine patches}

In 25], computations are performed locally in the Hermitian orthogonal complement of a point in projective space which Shub and Smale say can be considered as the tangent space of $\mathbb{P}^{N}$. To fix notation, assume that $x^{*} \in \mathbb{C}^{N+1} \backslash\{0\}$ such that $\left[x^{*}\right] \in \mathbb{P}^{N}$ is the current point in projective space under consideration. To avoid computations on vectors which are too large or too small, we will assume that $x^{*} \cdot x^{*}=\left\|x^{*}\right\|_{2}^{2}=1$. Thus, with this setup, the orthogonal affine patch is defined by

$$
x^{*} \cdot x=1 .
$$

If $y^{*} \in \mathbb{C}^{N+1}$ is the another point on this affine patch, let $\Delta x=y^{*}-x^{*}$. Thus, $\Delta x \cdot x^{*}=0$ so that $\Delta x$ is orthogonal to $x^{*}$ giving the method its name. See Figure 3(b) for a schematic view.

Example 3.3 Let $g(x)$ be as in (6) and $x^{*}=(1 / 2,-1 / 2,1 / 2,-1 / 2)$ so that $x^{*} \cdot x^{*}=1$ and $\left[x^{*}\right]=[1,-1,1,-1]$. Then, the condition number of the Jacobian matrix of $g$ with respect to the 2-norm with the affine patch defined by $x^{*} \cdot x=1$ is 4.37 . 
When path tracking, one uses an orthogonal affine coordinate patch based on the current point on the path and performs a predictor-corrector step in that patch. If the step is successful, the patch is updated based on the new point on the path (see Section 5).

The advantage of using an orthogonal affine coordinate patch is the typically well-controlled condition number. When using a fixed patch as in Section 3.1, one can globally remove a variable. With a locally adapting patch, one is able to locally remove a variable which will typically depend upon all of the other variables. The next method uses a locally adapted patch that fixes one variable.

\subsection{Coordinate-wise affine patches}

Coordinate-wise affine patches have the form $x_{j}=1$ for some $j \in\{0, \ldots, N\}$. For example, the points in (5) from Ex. 3.1 are represented using the coordinate-wise patch $x_{0}=1$. The advantage of using such a coordinate-wise patch is the simplicity of setting a coordinate equal to 1 . One disadvantage could be having the other coordinates be large if the point is "near" the hyperplane at "infinity," i.e., $x_{j}=0$. To overcome this, we locally adapt the selection of the coordinate $j$. That is, if $x^{*} \in \mathbb{C}^{N+1} \backslash\{0\}$ corresponds to $\left[x^{*}\right] \in \mathbb{P}^{N}$, we can assume that $\left\|x^{*}\right\|_{\infty}=1$ and select one coordinate $j$ such that $x_{j}^{*}=1$. Hence, the corresponding coordinate-wise affine patch is $e_{j} \cdot x=1$ where $e_{j}$ is the $j^{\text {th }}$ standard coordinate vector. See Figure $3(\mathrm{c})$ for a schematic view.

Example 3.4 Let $g(x)$ be as in (6) with $\left[x^{*}\right]=[1,-1,1,-1]$. The condition number of the $J a$ cobian matrix of $g$ with respect to the 2 -norm is 10.2 when using either $x_{0}=1$ or $x_{3}=1$ and 8.5 when using either $x_{1}=1$ or $x_{2}=1$.

As with the orthogonal patch in Section 3.2, when path tracking, we utilize a local strategy which updates the coordinate $j$ defining the affine patch after each successful step (see Section 5). Although the condition number is typically not as small as the orthogonal case, it is trivial to remove a variable since $x_{j}=1$ which helps to reduce the cost of linear algebra in taking a step.

\subsection{Optimal patching}

The affine patches described in Sections 3.13 .3 are of the form $v \cdot x=1$ for a vector $v \in \mathbb{C}^{N+1}$. Although one would like to minimize the condition number of the Jacobian over all such vectors $v \in \mathbb{C}^{N+1}$ for a given solution, we will efficiently approximate solving this large optimization problem by considering rescalings. The following demonstrates that this can yield improvements.

Example 3.5 Reconsider the setup from Ex. 3.4. Fix $z^{*}=(1,-1,1,-1)$ and $\alpha=e_{0}$. For $\lambda \in \mathbb{C} \backslash\{0\}$, consider $v=\lambda \cdot \alpha$ and $x^{*}=z^{*} / \lambda$ so that the patch is simply defined by $v \cdot x=\lambda \cdot x_{0}=1$, i.e., $x_{0}=1 / \lambda$. When $\lambda=1$, Ex. 3.4 showed that the condition number is 10.2 with Figure 4 showing the condition number as a function of $\lambda$. When $\lambda=1.7$, the condition number decreases to 7.2 .

Let $f(x)$ be a system of $N$ polynomials, each of degree $d>0$, defined on $\mathbb{P}^{N}, \alpha \in \mathbb{C}^{N+1} \backslash\{0\}$, $\lambda>0$, and affine patch $v \cdot x=1$ where $v=\lambda \cdot \alpha$. Suppose that $z^{*} \in \mathbb{C}^{N+1}$ with $\alpha \cdot z^{*}=1$ such that $\left[z^{*}\right] \in \mathbb{P}^{N}$ solves $f=0$. We aim to select the scaling $\lambda$ to improve the conditioning at $x^{*}=z^{*} / \lambda$. Since each $f_{i}$ is homogeneous of degree $d>0$, each entry of the gradient $\nabla f_{i}$ is either 0 


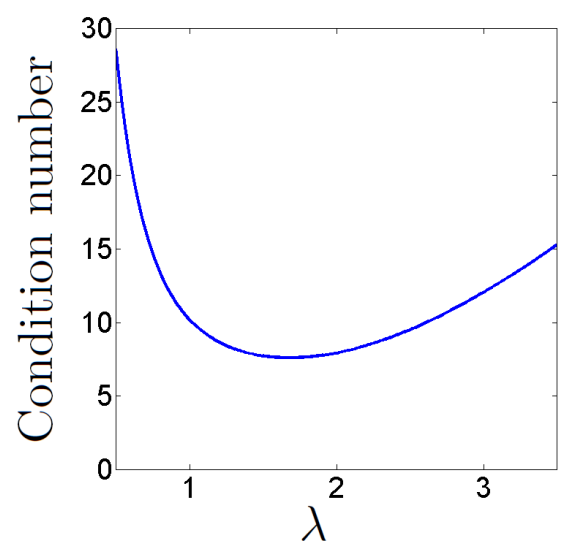

Figure 4: Plot of condition number as a function of the scaling parameter $\lambda$.

or homogeneous of degree $d-1$. Hence, consider the $(N+1) \times(N+1)$ matrix

$$
M(\lambda)=\lambda^{d-1}\left[\begin{array}{c}
\nabla f_{1}\left(z^{*} / \lambda\right) \\
\vdots \\
\nabla f_{N}\left(z^{*} / \lambda\right) \\
\lambda \cdot \alpha^{H}
\end{array}\right]=\left[\begin{array}{c}
\nabla f_{1}\left(z^{*}\right) \\
\vdots \\
\nabla f_{N}\left(z^{*}\right) \\
\lambda^{d} \cdot \alpha^{H}
\end{array}\right]=\left[\begin{array}{c}
J f\left(z^{*}\right) \\
\lambda^{d} \cdot \alpha^{H}
\end{array}\right]
$$

where $J f\left(z^{*}\right)$ is the Jacobian matrix of $f$ evaluated at $z^{*}$. To further simplify the computation, we consider minimizing $\kappa_{\infty, 1}(M(\lambda))=\|M(\lambda)\|_{\infty} \cdot\left\|M(\lambda)^{-1}\right\|_{1}$ which the following shows can be minimized using data from $M(1)$ and $M(1)^{-1}$.

Theorem 3.6 If $M(\lambda)$ from (7) is written as $M(\lambda)=\left[\begin{array}{c}J \\ \lambda^{d} \cdot \alpha^{H}\end{array}\right]$ and $M(1)^{-1}=\left[\begin{array}{ll}K & \beta\end{array}\right]$ where $J \in \mathbb{C}^{N \times(N+1)}, K \in \mathbb{C}^{(N+1) \times N}$, and $\alpha, \beta \in \mathbb{C}^{N}$, then $\kappa_{\infty, 1}(M(\lambda))$ is minimized when

$$
\lambda=\sqrt[2 d]{\frac{\|J\|_{\infty} \cdot\|\beta\|_{1}}{\|K\|_{1} \cdot\|\alpha\|_{1}}} .
$$

Proof. From $M(1)^{-1}$, it is easy to verify that $M(\lambda)^{-1}=\left[\begin{array}{ll}K & \beta / \lambda^{d}\end{array}\right]$. Hence,

$$
\|M(\lambda)\|_{\infty}=\max \left\{\|J\|_{\infty}, \lambda^{d} \cdot\|\alpha\|_{1}\right\} \quad \text { and } \quad\left\|M(\lambda)^{-1}\right\|_{1}=\max \left\{\|K\|_{1},\|\beta\|_{1} / \lambda^{d}\right\}
$$

so that

$$
\kappa_{\infty, 1}(M(\lambda))=\max \left\{\|J\|_{\infty} \cdot\|\beta\|_{1} / \lambda^{d},\|J\|_{\infty} \cdot\|K\|_{1},\|\alpha\|_{1} \cdot\|\beta\|_{1}, \lambda^{d} \cdot\|K\|_{1} \cdot\|\alpha\|_{1}\right\} .
$$

Hence, $\kappa_{\infty, 1}(M(\lambda))$ is a convex function such that $\kappa_{\infty, 1}(M(\lambda))=\|J\|_{\infty} \cdot\|\beta\|_{1} / \lambda^{d}$ for $0<\lambda \ll 1$ and $\kappa_{\infty, 1}(M(\lambda))=\lambda^{d} \cdot\|\alpha\|_{1} \cdot\|K\|_{1}$ for $\lambda \gg 1$. Figure 5 presents an example plot of $\kappa_{\infty, 1}(M(\lambda))$. In particular, we have that the minimum is achieved when

$$
\|J\|_{\infty} \cdot\|\beta\|_{1} / \lambda^{d}=\lambda^{d} \cdot\|\alpha\|_{1} \cdot\|K\|_{1}
$$

which occurs when $\lambda=\sqrt[2 d]{\frac{\|J\|_{\infty} \cdot\|\beta\|_{1}}{\|K\|_{1} \cdot\|\alpha\|_{1}}}$. In particular, the minimum of $\kappa_{\infty, 1}$ is

$$
\max \left\{\sqrt{\|J\|_{\infty} \cdot\|K\|_{1} \cdot\|\alpha\|_{1} \cdot\|\beta\|_{1}},\|J\|_{\infty} \cdot\|K\|_{1},\|\alpha\|_{1} \cdot\|\beta\|_{1}\right\} .
$$




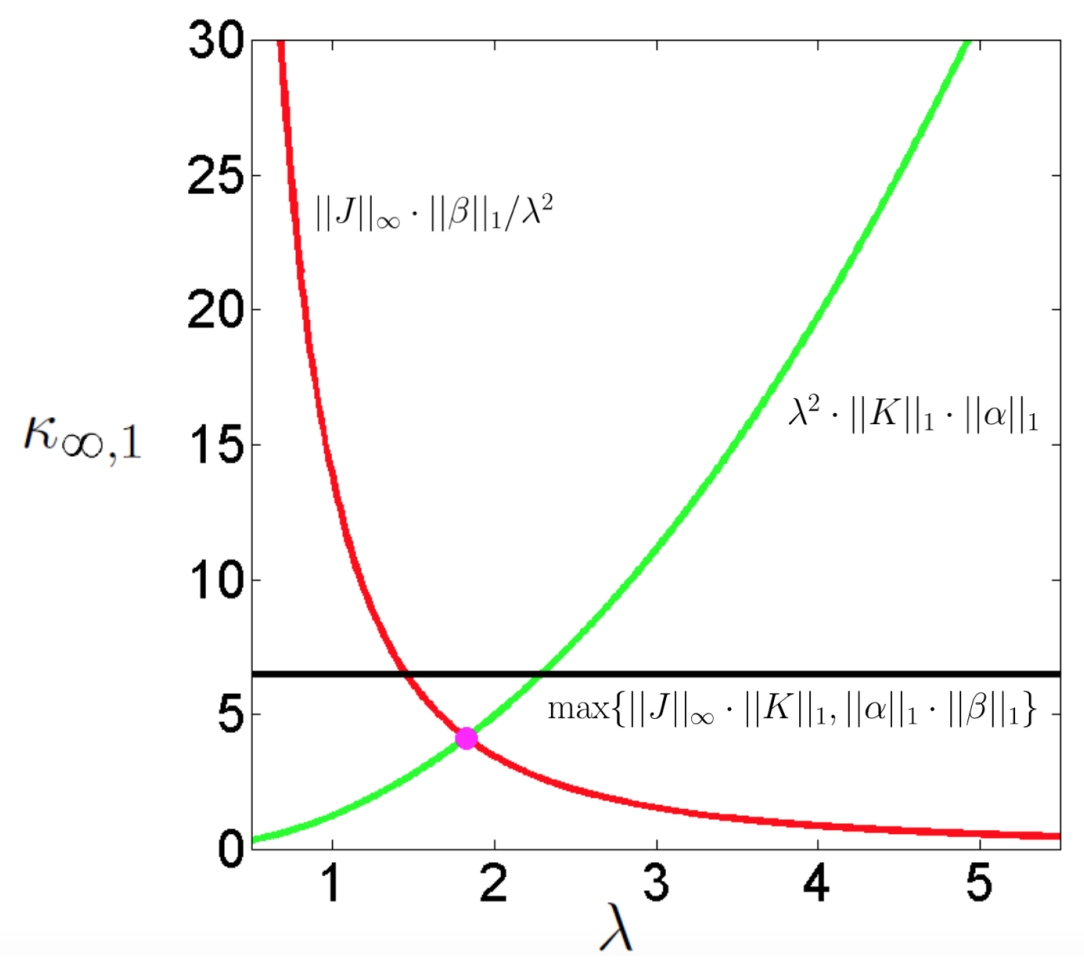

Figure 5: Plot of $\kappa_{\infty, 1}$ a function of the scaling parameter $\lambda$.

Example 3.7 To illustrate Theorem 3.6, consider the polynomial system

$$
f(x)=\left[\begin{array}{c}
x_{0} x_{2}-x_{1}^{2} \\
x_{0}^{2}+x_{1}^{2}+x_{2}^{2}-x_{3}^{2} \\
x_{1} x_{2}+x_{1} x_{3}-x_{0}^{2}
\end{array}\right]
$$

using the coordinate-wise patch $x_{3}=1$ which is defined by $\alpha=(0,0,0,1)$ with solution

$$
z^{*}=((\sqrt{5}+1) / 4,1 / 2,(\sqrt{5}-1) / 4,1) \approx(0.8090,0.5,0.3090,1) .
$$

Following the notation of Thm. 3.6, we have

$$
d=2, \quad\|J\|_{\infty}=5.2361, \quad\|K\|_{1}=1.2361, \quad\|\alpha\|_{1}=1, \quad \text { and }\|\beta\|_{1}=2.6180
$$

so that

$$
\lambda=\sqrt[4]{\frac{\|J\|_{\infty} \cdot\|\beta\|_{1}}{\|K\|_{1} \cdot\|\alpha\|_{1}}} \approx 1.8249
$$

is the scaling factor to minimize $\kappa_{\infty, 1}$ as shown in Figure 5 . Hence, we take the affine patch defined by $v \cdot x=1$ where $v=\lambda \cdot \alpha \approx(0,0,0,1.8249)$ yielding the corresponding point

$$
x^{*}=z^{*} / \lambda \approx(0.4433,0.2740,0.1693,0.5480) \text {. }
$$

When using an orthogonal patch, the following shows that we can simplify the computation of $\lambda$. 
Corollary 3.8 Using the same setup as Theorem 3.6, if $\alpha=z^{*}$ with $\alpha^{H} \alpha=\|\alpha\|_{2}^{2}=1$, then $\beta=\alpha=z^{*}$ so that $\kappa_{\infty, 1}(M(\lambda))$ is minimized when

$$
\lambda=\sqrt[2 d]{\frac{\|J\|_{\infty}}{\|K\|_{1}}} .
$$

Proof. Following the notation from (7) and Thm. 3.6. $M(1)=\left[\begin{array}{c}J \\ \alpha^{H}\end{array}\right]$ and $M(1)^{-1}=\left[\begin{array}{ll}K & \beta\end{array}\right]$. Euler's Theorem yields that $J \alpha=0$ since each $f_{i}$ is homogeneous and $f\left(z^{*}\right)=0$. Since $\beta$ is the unique vector such that $J \beta=0$ and $\alpha^{H} \beta=1$, we have $\alpha=\beta$ and the result follows from Thm. 3.6.

Example 3.9 Reconsider $f$ from Ex. 3.7 with the orthogonal patch $\alpha \cdot x=1$ where $\alpha=z^{*}$ and

$$
z^{*}=((\sqrt{5}+1) / \sqrt{32}, 1 / \sqrt{8},(\sqrt{5}-1) / \sqrt{32}, 1 / \sqrt{2}) \approx(0.5721,0.3536,0.2185,0.7071) .
$$

By Cor. 3.8, $\alpha=\beta$ and we have

$$
d=2, \quad\|J\|_{\infty}=3.7025, \text { and }\|K\|_{1}=1.7481
$$

so that

$$
\lambda=\sqrt[4]{\frac{\|J\|_{\infty}}{\|K\|_{1}}} \approx 1.2064
$$

is the scaling factor to minimize $\kappa_{\infty, 1}$. Hence, we take the affine patch defined by $v \cdot x=1$ where $v=\lambda \cdot \alpha \approx(0.6901,0.4265,0.2636,0.8530)$ yielding the corresponding point

$$
x^{*}=z^{*} / \lambda \approx(0.4742,0.2931,0.1811,0.5861) .
$$

Figure 6 compares $\kappa_{\infty, 1}(M(\lambda))$ with the condition number $\kappa_{2}(M(\lambda))$.

\section{Randomization}

The polynomial system $f(x)$ in Ex. 3.1 is overdetermined since it consists of 4 polynomials defined on $\mathbb{P}^{3}$. If one considers appending the patch equation $x_{0}-1=0$ to $f(x)$, the system is still overdetermined with 5 polynomials defined on $\mathbb{C}^{4}$. The first 3 polynomials define the twisted cubic curve, which has codimension 2, while the fourth polynomial is a hyperplane that intersects the twisted cubic curve transversely. Since a general perturbation applied to the first 3 polynomials results in a system with no solutions, numerically solving inexact overdetermined systems is unstable, e.g., see $[4, \S 9.2]$. One could recover stability by using Gauss-Newton least-squares approaches, e.g., see [7]. Another approach for stabilization is to replace overdetermined systems with wellconstrained subsystems. The following is a version of Bertini's Theorem, e.g., see [28, Thm. A.8.7] and [4, Thm. 9.3], which permits such replacements using well-constrained subsystems.

Theorem 4.1 (Bertini's Theorem) If $f(x)$ is a system of $n$ polynomials on $\mathbb{C}^{N}$ and $1 \leqslant k \leqslant n$, then there exists a Zariski open dense set $U \subset \mathbb{C}^{k \times n}$ such that for every $A \in U$, each generically nonsingular irreducible component of the solution set of $f=0$ of codimension at most $k$ is a generically nonsingular irreducible component of the solution set of $A \cdot f=0$. 


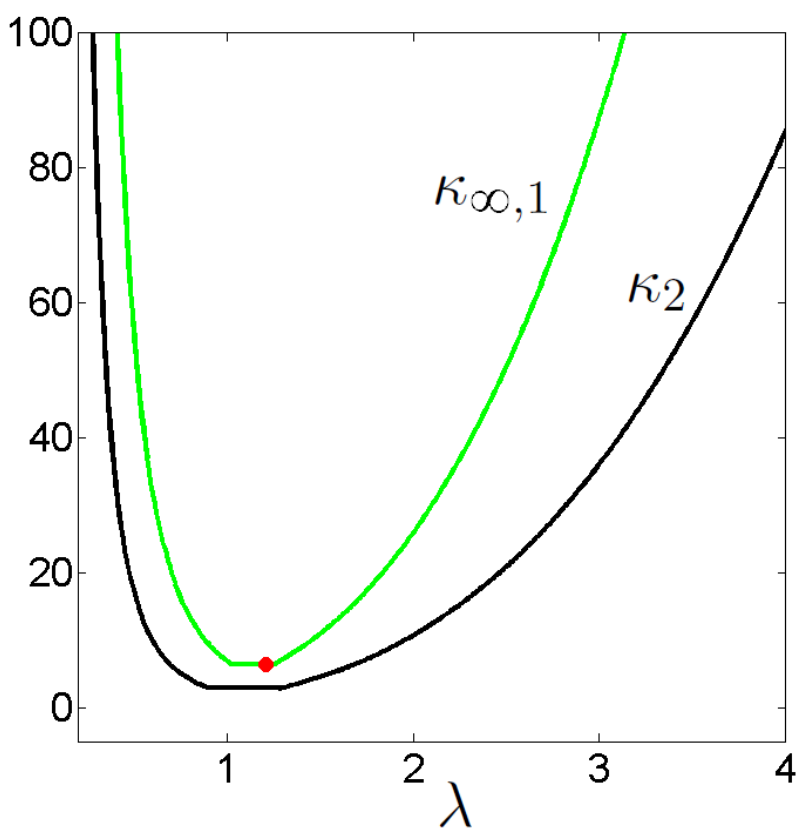

Figure 6: Plot of $\kappa_{\infty, 1}$ and $\kappa_{2}$ as a function of the scaling parameter $\lambda$.

We state Bertini's Theorem to focus on generically nonsingular irreducible components for path tracking purposes (see Section 5). One can always reduce to this case using deflation, e.g., 11, 20]. We note that randomization could also add extra solutions which do not solve the original system. Such extraneous solutions can be certifiably identified [10].

Example 4.2 For $f(x)$ as in (4) together with the patch equation $x_{0}-1=0$, consider the system

$$
g(x)=\left[\begin{array}{ccccc}
2 & -1 & -3 & 2 & 2 \\
-2 & -1 & 0 & 3 & -4 \\
5 & 3 & -1 & -2 & -4 \\
-5 & 3 & 2 & 2 & 0
\end{array}\right] \cdot\left[\begin{array}{c}
f(x) \\
x_{0}-1
\end{array}\right]=0
$$

which is a well-constrained system consisting of 4 polynomial equations on $\mathbb{C}^{4}$. Bertini's Theorem yields that the three points corresponding to (5) are isolated nonsingular solutions to $g=0$. Randomization has also added two additional solutions to $g=0$, approximately

$$
(0.7955 \pm 0.0744 i, 0.3755 \mp 0.6315 i,-1.2239 \mp 0.1598 i,-0.6730+0.9810 i)
$$

where $i=\sqrt{-1}$ which are easily identified since $x_{0} \neq 1$.

In (8), we selected the randomizing matrix $A$ to have small integer entries for presentation purposes. In practice, the matrix $A$ is selected to have random complex entries.

Analogous to the patching strategies described in Section 3, we describe three randomization strategies. The first is based directly on Theorem 4.1 which utilizes a fixed randomization matrix, a commonly used technique in numerical algebraic geometry computations, e.g., [4, § 9.2]. The second utilizes a locally adapted orthogonalization strategy based on the Moore-Penrose pseudoinverse. To create sparse randomizations, the third utilizes a locally adapted leverage score strategy. 


\subsection{Fixed randomization}

As described in Bertini's Theorem (Theorem 4.1), one can utilize a fixed general randomization matrix $A \in \mathbb{C}^{k \times n}$ to yield a well-constrained system. As detailed in $[28, \S 13.5]$, one could take $A$ to have the form $A=[I Q]$ where $I$ is the $k \times k$ identity matrix and $Q \in \mathbb{C}^{k \times(n-k)}$ is general to reduce the number of computations needed to randomize the system.

Similar to the fixed affine coordinate patch in Section 3.1, the disadvantage of using a fixed randomization is that ill-conditioning can artificially be introduced as demonstrated in the following.

Example 4.3 With $f(x)$ as in (4), consider the system $h: \mathbb{C}^{4} \rightarrow \mathbb{C}^{5}$ where

$$
h(x)=\left[\begin{array}{c}
f(x) \\
x_{0}-1
\end{array}\right]
$$

and isolated nonsingular solution $(1,-1,1,-1)$. Table 2 shows the condition number with respect to the 2-norm of the Jacobian matrix for various randomizations of the form $\left[\begin{array}{l}I \\ Q\end{array}\right] \cdot h$ where $I$ is the $4 \times 4$ identity matrix and $Q \in \mathbb{C}^{4 \times 1}$ at this isolated nonsingular solution.

\begin{tabular}{c|c|c|c}
$Q$ & {$[1,1,1,1]^{T}$} & {$[-0.0109,0.5208,0.4013,0.7534]^{T}$} & {$[-0.0889,0.6266,0.7152,0.2966]^{T}$} \\
\hline $\mathrm{CN}$ & 33.3 & 185.6 & $67,193.2$
\end{tabular}

Table 2: Condition number of the Jacobian matrix of $[I Q] \cdot h$ for different choices of $Q$

One can attempt to limit this artificial ill-conditioning by using a randomization which is locally adapted to the current solution under consideration. The next two subsections consider two methods for selecting local randomizations.

\subsection{Pseudoinverse randomization}

At a nonsingular solution, the Jacobian matrix has full rank so that one can use the Moore-Penrose pseudoinverse to construct a randomization matrix. To that end, assume that $f: \mathbb{C}^{N} \rightarrow \mathbb{C}^{n}$ is a polynomial system and $x^{*} \in \mathbb{C}^{N}$ is an isolated nonsingular solution of $f=0$, i.e., $f\left(x^{*}\right)=0$ and rank $J f\left(x^{*}\right)=N \leqslant n$ where $J f\left(x^{*}\right)$ is the Jacobian matrix of $f$ evaluated at $x^{*}$. Via the singular value decomposition, we can find unitary matrices $U \in \mathbb{C}^{n \times N}$ and $V \in \mathbb{C}^{N \times N}$ and invertible diagonal matrix $\Sigma \in \mathbb{R}^{N \times N}$ such that

$$
J f\left(x^{*}\right)=U \cdot \Sigma \cdot V^{H} \in \mathbb{C}^{n \times N}
$$

where $V^{H}$ is the Hermitian (conjugate) transpose of $V$. The Moore-Penrose pseudoinverse of $J f\left(x^{*}\right)$ is $J f\left(x^{*}\right)^{\dagger}=V \cdot \Sigma^{-1} \cdot U^{H} \in \mathbb{C}^{N \times n}$ so that

$$
J f\left(x^{*}\right)^{\dagger} \cdot J f\left(x^{*}\right)=I
$$

where $I$ is the $N \times N$ identity matrix. Therefore, the randomized well-constrained subsystem

$$
g(x)=J f\left(x^{*}\right)^{\dagger} \cdot f(x)
$$

has a nonsingular solution at $x^{*}$ with $J g\left(x^{*}\right)=I$. 
Example 4.4 With $h: \mathbb{C}^{4} \rightarrow \mathbb{C}^{5}$ as in (9) and $x^{*}=(1,-1,1,-1)$, we have

$$
J h\left(x^{*}\right)^{\dagger}=\left[\begin{array}{ccccc}
0 & 0 & 0 & 0 & 1 \\
1 / 6 & 1 / 3 & 1 / 6 & 1 / 2 & -1 \\
1 / 3 & -1 / 3 & -2 / 3 & -1 & 1 \\
-1 / 2 & 0 & 1 / 2 & 3 / 2 & -1
\end{array}\right] .
$$

Thus, for the randomized well-constrained subsystem $g(x)=J h\left(x^{*}\right)^{\dagger} \cdot h(x), g\left(x^{*}\right)=0$ and $J g\left(x^{*}\right)=$ $I \in \mathbb{C}^{4 \times 4}$.

When path tracking, one uses the pseudoinverse based on the current point on the path and performs a predictor-corrector step with that randomized system. If the step is successful, the randomization is updated based on the new point on the path (see Section 5).

The advantage of using the pseudoinverse randomization is that the Jacobian matrix at the current point of the randomized system is the identity matrix, a perfectly conditioned matrix. The disadvantage is the extra computations: both in the computation of the pseudoinverse and to utilize the randomization which is typically dense. The following uses a sparse randomization.

\subsection{Leverage score randomization}

The randomizations in Sections 4.1 and 4.2 construct a new system which typically depends upon all of the polynomials in the original system. We aim to design an approach to select a well-constrained subset which has a nonsingular solution at the current point. For example, if the given system is vastly overdetermined, we aim to select a subset of polynomials from the system rather than having to randomize together all of the polynomials. The method that we propose is based on leverage scores which were originally used to find outliers in data when computing regression analysis [13]. We follow the approach in [14 which states that leverage scores can be used to describe important data in a matrix. In our case, we aim to locate polynomials in the system whose gradients are important rows of the Jacobian matrix evaluated at the given point.

Definition 4.5 (Leverage scores) For a matrix $M \in \mathbb{C}^{m \times n}$ of rank $n \leqslant m$, let $Q \in \mathbb{C}^{m \times n}$ be any unitary matrix whose columns form a basis for the column span of $M$. Then, the leverage scores $\ell_{1}, \ldots, \ell_{m} \in \mathbb{R}_{\geqslant 0}$ for $M$ are defined by

$$
\ell_{j}=\left\|Q_{j}\right\|_{2}^{2}
$$

where $Q_{j}$ is the $j^{\text {th }}$ row of $Q$.

The definition of leverage scores is basis independent $[14, \S 5.1]$ so that that leverage scores are well-defined. Moreover, since $Q$ is unitary, each $\ell_{j} \in[0,1]$ with $\sum_{j=1}^{m} \ell_{j}=n$.

As above, assume that $f: \mathbb{C}^{N} \rightarrow \mathbb{C}^{n}$ is a polynomial system and $x^{*} \in \mathbb{C}^{N}$ is an isolated nonsingular solution of $f=0$. Rather than perform a singular value decomposition on $J f\left(x^{*}\right)$ as in Section 4.2, we perform a (column pivoted) QR factorization of $J f\left(x^{*}\right)$, that is, we compute

$$
J f\left(x^{*}\right)=Q \cdot R \cdot P
$$

where $Q \in \mathbb{C}^{n \times N}$ is unitary, $R \in \mathbb{C}^{N \times N}$ is upper triangular and $P \in \mathbb{R}^{N \times N}$ is a permutation matrix. The permutation matrix swaps the columns which corresponds with simply reordering the variables. With this setup, we construct the randomization matrix iteratively based on the largest values of the leverage scores of $J f\left(x^{*}\right)$ as follows. 
Definition 4.6 (Leverage score randomization matrix) Following the setup as above, suppose that $\ell_{1}, \ldots, \ell_{n}$ are the leverage scores of $J f\left(x^{*}\right)$. Construct a reordering of the leverage scores so that they are in decreasing order, say $\ell_{k_{1}} \geqslant \ell_{k_{2}} \geqslant \cdots \geqslant \ell_{k_{n}} \geqslant 0$. Fix $j_{1}=1$. For $1 \leqslant r<N$, given $j_{1}<\cdots<j_{r}$, select $j_{r+1}>j_{t}$ to be the minimum value such that the $k_{j_{1}}, \ldots, k_{j_{r+1}}$ rows of $J f\left(x^{*}\right)$ are linearly independent. The corresponding leverage score randomization matrix is $A \in \mathbb{R}^{N \times n}$ which has the following $N$ nonzero entries:

$$
A_{r, k_{j_{r}}}=\left\|J f\left(x^{*}\right)_{k_{j_{r}}}\right\|_{2}^{-1} \text { for } r=1, \ldots, N
$$

where $J f\left(x^{*}\right)_{p}$ is the $p^{\text {th }}$ row of $J f\left(x^{*}\right)$.

Example 4.7 To illustrate, consider $x^{*}=(1,1)$ and

$$
f(x)=\left[\begin{array}{l}
x_{1}-1 \\
x_{1}-1 \\
x_{2}-1 \\
x_{2}-1
\end{array}\right] \text { so that } J f\left(x^{*}\right)=\left[\begin{array}{ll}
1 & 0 \\
1 & 0 \\
0 & 1 \\
0 & 1
\end{array}\right]=\left[\begin{array}{cc}
1 / \sqrt{2} & 0 \\
1 / \sqrt{2} & 0 \\
0 & 1 / \sqrt{2} \\
0 & 1 / \sqrt{2}
\end{array}\right]\left[\begin{array}{cc}
\sqrt{2} & 0 \\
0 & \sqrt{2}
\end{array}\right] \text {. }
$$

The leverage scores of $J f\left(x^{*}\right)$ are all equal, namely $\ell_{1}=\cdots=\ell_{4}=1 / 2$. With the trivial ordering $k_{j}=j$, Definition 4.6 produces $j_{1}=1$ and $j_{2}=3$ since the first and second rows of $J f\left(x^{*}\right)$ are not linearly independent. Since each row of $J f\left(x^{*}\right)$ has norm 1 , the leverage score randomized matrix is

$$
A=\left[\begin{array}{llll}
1 & 0 & 0 & 0 \\
0 & 0 & 1 & 0
\end{array}\right] \quad \text { with } \quad A \cdot f(x)=\left[\begin{array}{l}
x_{1}-1 \\
x_{2}-1
\end{array}\right] \text {. }
$$

Proposition 4.8 With the setup described above, if $A$ is a leverage score randomization matrix for $J f\left(x^{*}\right)$, then $A \cdot J f\left(x^{*}\right)$ has rank $N$ such that each row has unit length in the 2-norm.

Proof. Since $J f\left(x^{*}\right)$ has rank $N$, matrix $Q$ in 10$)$ has rank $N$. In particular, at least $N$ rows of both $J f\left(x^{*}\right)$ and $Q$ must be nonzero with $\ell_{k_{1}}>0$. Moreover, since $R \cdot P$ is invertible, $J f\left(x^{*}\right)_{j}=0$ if and only if $Q_{j}=0$, i.e., $\ell_{j}=0$. Thus, if $j_{1}, \ldots, j_{N}$ are selected as in Defn. 4.6, then $\left\|J f\left(x^{*}\right)_{k_{j_{r}}}\right\|_{2}>0$ for $r=1, \ldots, N$. Hence, it follows that the rows of $A \cdot J f\left(x^{*}\right)$ have unit length in the the 2-norm.

The fact that the resulting matrix $A \cdot J f\left(x^{*}\right)$ has rank $N$ using such a greedy selection of rows is classical in linear algebra and follows from the dimension of the row span of $J f\left(x^{*}\right)$ being $N$.

Example 4.9 With $h(x)$ as in (9) and $x^{*}=(1,-1,1,-1)$, the leverage scores of Jh( $\left.x^{*}\right)$ are $\ell_{1}=\ell_{2}=\ell_{3}=2 / 3$ and $\ell_{4}=\ell_{5}=1$. Regarding leverage scores as a measure of importance, this shows that the fourth and fifth polynomials in $h$, namely $x_{0}+x_{1}+x_{2}+x_{3}$ and $x_{0}-1$, respectively, are equally the two most important at $x^{*}$. The other three polynomials which define the twisted cubic are equally important to each other, but less than the two linear polynomials. By taking the reordering $k_{1}=4, k_{2}=5, k_{3}=1, k_{4}=2$, and $k_{5}=3$, we have the randomized system

$$
g(x)=\left[\begin{array}{ccccc}
0 & 0 & 0 & 1 / 2 & 0 \\
0 & 0 & 0 & 0 & 1 \\
1 / \sqrt{6} & 0 & 0 & 0 & 0 \\
0 & 1 / 2 & 0 & 0 & 0
\end{array}\right] \cdot h(x)=\left[\begin{array}{c}
\left(x_{0}+x_{1}+x_{2}+x_{3}\right) / 2 \\
x_{0}-1 \\
\left(x_{0} x_{2}-x_{1}^{2}\right) / \sqrt{6} \\
\left(x_{1} x_{2}-x_{0} x_{3}\right) / 2
\end{array}\right]
$$

so that each row of

$$
J g\left(x^{*}\right)=\left[\begin{array}{cccc}
1 / 2 & 1 / 2 & 1 / 2 & 1 / 2 \\
1 & 0 & 0 & 0 \\
1 / \sqrt{6} & 2 / \sqrt{6} & 1 / \sqrt{6} & 0 \\
1 / 2 & 1 / 2 & -1 / 2 & -1 / 2
\end{array}\right]
$$

has unit length in the 2-norm and its condition number with respect to the 2 -norm is 8.8 . 
Similar to the pseudoinverse randomization when path tracking, one uses the leverage score randomization based on the current point on the path and performs a predictor-corrector step with that randomized system. If the step is successful, the randomization is updated based on the new point on the path (see Section 5).

The advantage of using a leverage score randomization is that the randomizing matrix is sparse and selects a well-constrained subset of the original polynomials. Thus, one saves computational time by only evaluating the polynomials and their gradients of the polynomials which are selected.

\section{$5 \quad$ Path tracking algorithms}

We now aim to incorporate the patching and randomization strategies into path tracking. Suppose that $F(x ; p)$ is a parameterized system which is polynomial in the variables $x \in X$ and analytic in the parameters $p \in \mathbb{C}^{P}$. Depending on the structure of $F$, we may regard $X$ as a projective or affine space, or, more generally, as a product of such spaces. Suppose that $p^{*} \in \mathbb{C}^{P}$ is generic and $S\left(p^{*}\right)$ consists of the isolated nonsingular solutions of $F\left(x ; p^{*}\right)=0$. As mentioned in Section 2, there is a generic behavior of $F$ with respect to the parameter space $\mathbb{C}^{P}$ so that, for given $\hat{p} \in \mathbb{C}^{P}$, we can use the parameter homotopy $H$ defined in (2) with start points $S\left(p^{*}\right)$ at $t=1$ to compute $S(\hat{p})$, the set of all nonsingular isolated solutions of $F(x ; \hat{p})=0$. In particular, for each $x^{*} \in S\left(p^{*}\right)$, there is a smooth homotopy path $x(t)$ for $t \in(0,1]$ such that $x(1)=x^{*}$ and $H(x(t), t) \equiv 0$. Since $x(t)$ could be defined on products of projective and affine spaces, and $F$ could be overdetermined, we utilize patching and randomization strategies to track $x(t)$. To avoid having to deal with paths with divergent and singular endpoints, which can be handled using endgames [28, Chap. 10], we assume that $x(t)$ exists and is smooth on $[0,1]$ in Algorithm 1. By working intrinsically on the affine patch, one could attempt to reduce the linear algebra cost of performing a predictor-corrector step.

The justification for Algorithm 1 follows from Bertini's Theorem (Theorem 4.1) and the use of affine coordinate patches which permits computations regarding the path $x(t)$ to be performed using a well-constrained subsystem on an affine space. By having local control on the condition number, we aim to perform fewer operations when path tracking as exemplified in Section 7 .

Example 5.1 To illustrate Algorithm 1, we consider the parameterized system

$$
F(x ; p)=\left[\begin{array}{c}
x_{0} x_{2}-x_{1}^{2} \\
x_{1} x_{2}-x_{0} x_{3} \\
x_{1} x_{3}-x_{2}^{2} \\
x_{2}+p_{1} x_{0}+p_{2} x_{1}+p_{3} x_{3}
\end{array}\right]
$$

where $x \in X=\mathbb{P}^{3}$ and $p \in \mathbb{C}^{3}$. Thus, $F(x ; p)=0$ defines the intersection of the twisted cubic with a parameterized family of hyperplanes which clearly has 3 nonsingular isolated solutions generically. In the following, we consider tracking the 3 solution paths as $p^{*}=(1,1,1)$ deforms to $\hat{p}=(-1,0.1 i, 0)$, where $i=\sqrt{-1}$, starting with the 3 points in (5). This setup ensures that we will need to use an affine patch followed by a randomization.

For the fixed general affine patch, we used the randomly selected patch

$$
(0.3509+0.1476 i) x_{0}+(0.4524-0.4487 i) x_{1}-(0.4159+0.2470 i) x_{2}+(0.4609+0.0523 i) x_{3}=1
$$

where $i=\sqrt{-1}$. 


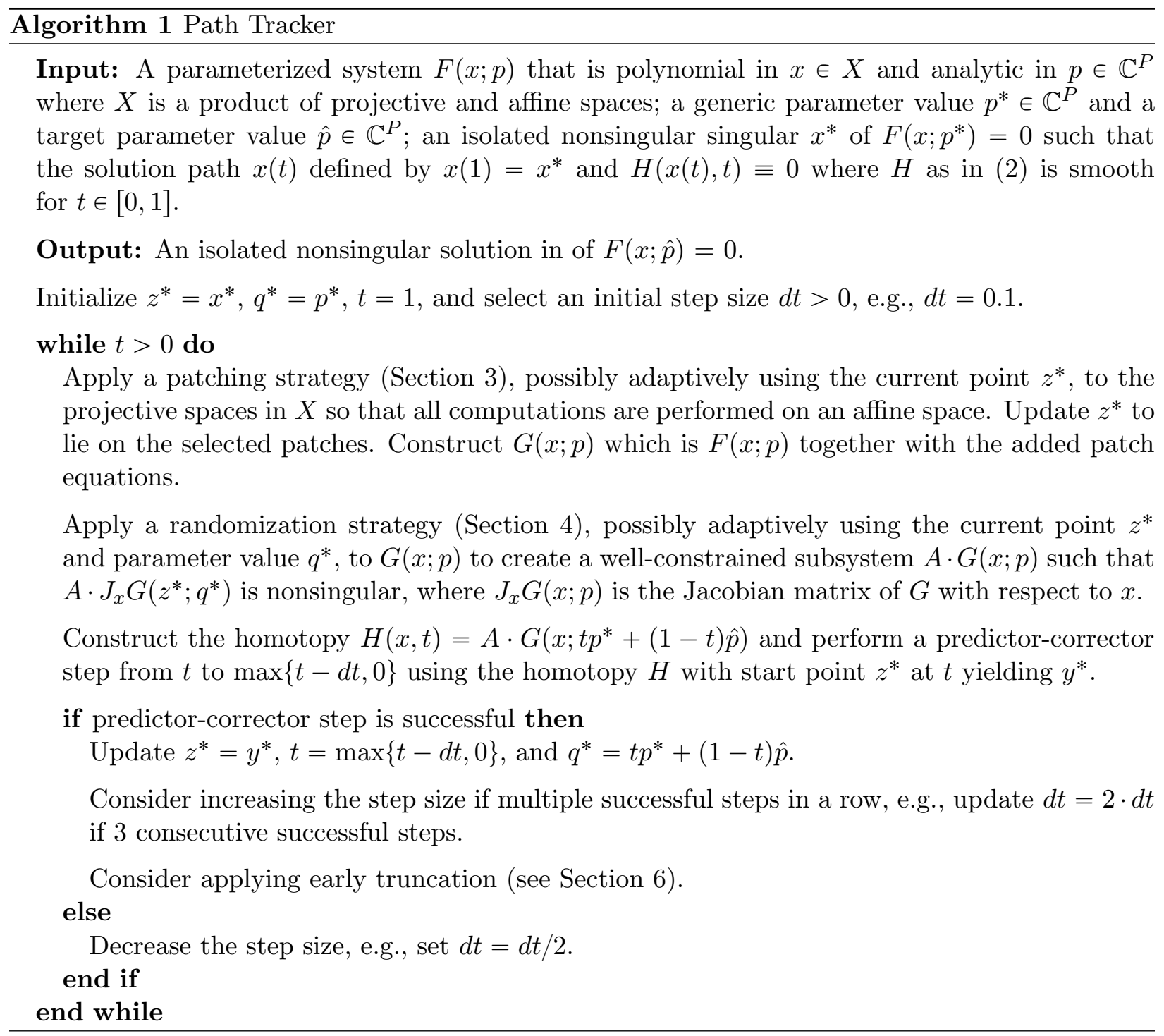


In Figures 17, 8, and 9, we compare the condition number with respect to the 2-norm of the Jacobian matrix along the three paths using the different patching and randomization strategies. For the fixed randomization, we used $A=\left[\begin{array}{ll}I & Q\end{array}\right] \in \mathbb{C}^{4 \times 5}$ with the randomly selected

$$
Q=[0.1792-0.1432 i,-0.7159-0.5784 i, 0.1866-0.4692 i, 0.4524+0.9864 i]^{T} .
$$

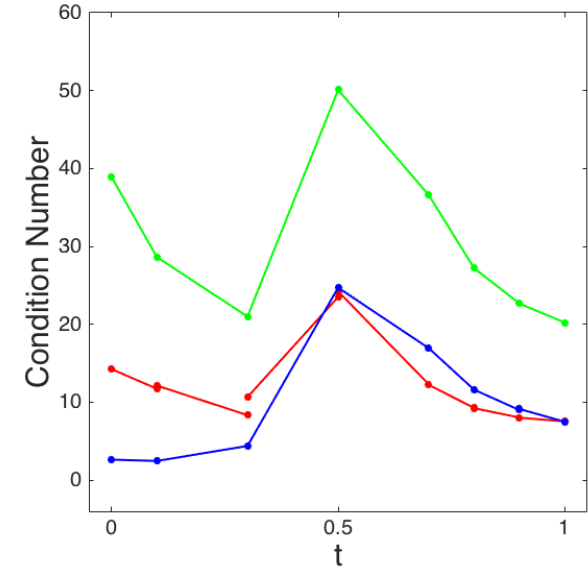

(a)

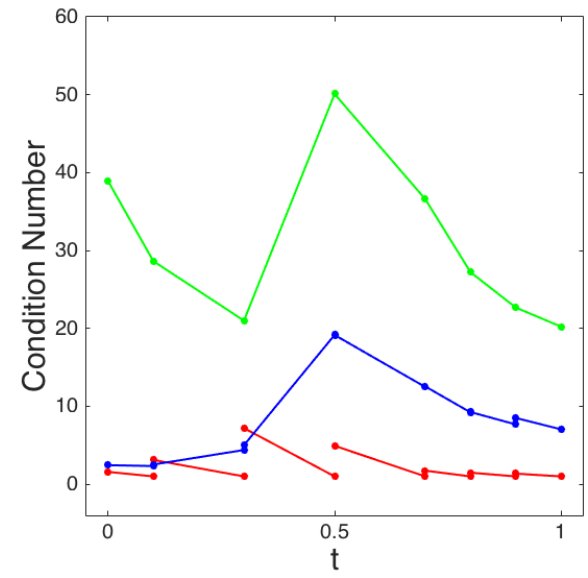

(b)

Figure 7: Plot of the condition numbers along the first path. (a) Using a fixed randomization, Green: fixed affine patch, Red: orthogonal affine patch, Blue: coordinate-wise affine patch. (b) Green: fixed randomization with fixed affine patch, Red: pseudoinverse randomization with orthogonal affine patch, Blue: leverage score randomization with coordinate-wise affine patch.

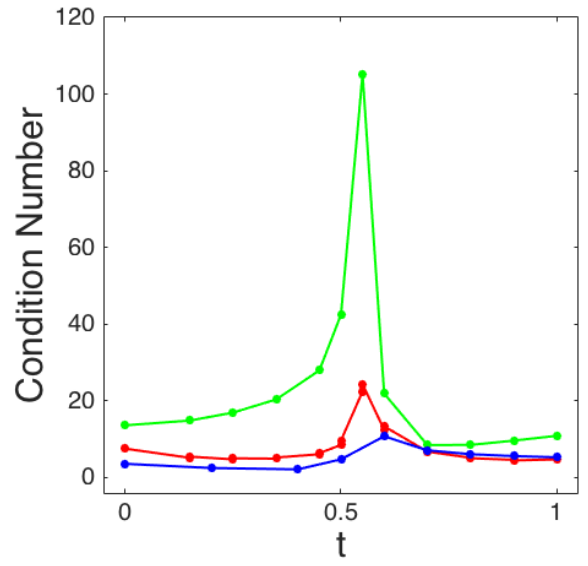

(a)

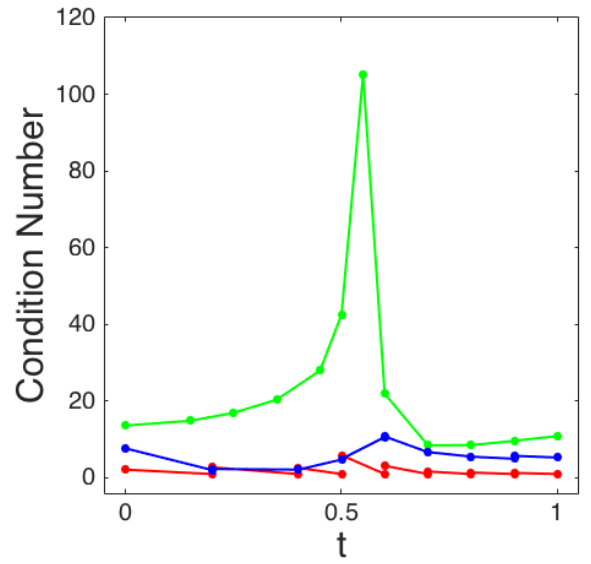

(b)

Figure 8: Plot of the condition numbers along the second path. (a) Using a fixed randomization, Green: fixed affine patch, Red: orthogonal affine patch, Blue: coordinate-wise affine patch. (b) Green: fixed randomization with fixed affine patch, Red: pseudoinverse randomization with orthogonal affine patch, Blue: leverage score randomization with coordinate-wise affine patch. 


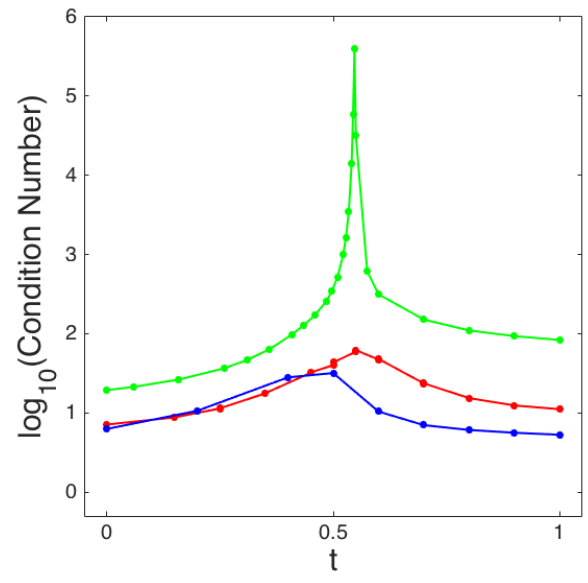

(a)

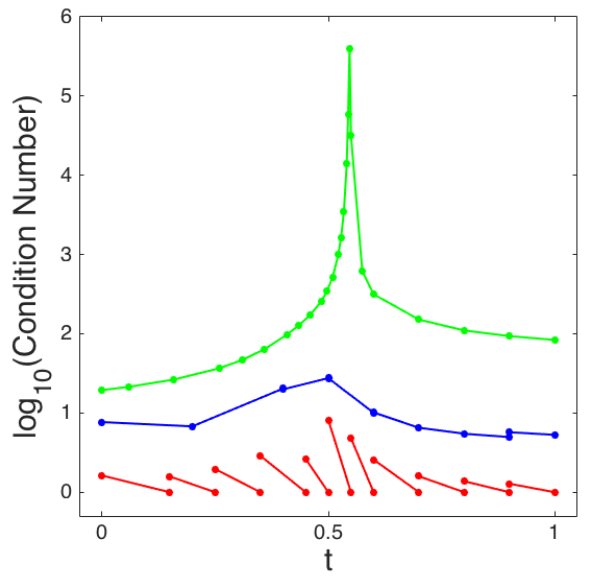

(b)

Figure 9: Plot of the logarithm of the condition numbers along the third path. (a) Using a fixed randomization, Green: fixed affine patch, Red: orthogonal affine patch, Blue: coordinate-wise affine patch. (b) Green: fixed randomization with fixed affine patch, Red: pseudoinverse randomization with orthogonal affine patch, Blue: leverage score randomization with coordinate-wise affine patch.

\section{Truncation}

In many applications, one is interested in computing real solutions. For a parameterized system, we are typically deforming from a complex parameter value $p^{*}$ to a real parameter value $\hat{p}$ and we would like a heuristic approach that could help identify which paths are headed to nonreal endpoints. The paths with (potentially) nonreal endpoints will be truncated to limit wasted computation.

The idea of our proposed test is to consider how the size of the imaginary part of the points on the path is changing with respect to $t$. Since real endpoints have imaginary part equal to 0 , we want to make a heuristic decision based on the data along the path to decide if the imaginary part could reasonably limit to 0 . If this is not reasonable, then we consider the path to be heading towards a nonreal endpoint. There is a trade-off between when to start applying this test. If the test is applied far from $t=0$, then the imaginary part will be significantly impacted by the starting parameter $p^{*}$. If the test is applied very close to $t=0$, then there is little computational savings in truncation. In our experiments, we start testing when $t<0.3$. See Algorithm 1 for the location of truncation in the path tracking algorithm.

Our proposed truncation test takes as input two points along the path, say $x\left(t_{1}\right)$ and $x\left(t_{2}\right)$ where $0<t_{2}<t_{1}$ and makes a decision based on the angle between the following two lines:

- line connecting $\left(t_{1},\left\|\operatorname{imag} x\left(t_{1}\right)\right\|_{2}\right)$ and $\left(t_{2},\left\|\operatorname{imag} x\left(t_{2}\right)\right\|_{2}\right)$, and

- line connecting $\left(t_{2},\left\|\operatorname{imag} x\left(t_{2}\right)\right\|_{2}\right)$ and $(0,0)$

as shown in Figure 10. A large angle suggests that it is realistic to believe that the path is heading towards a nonreal endpoint. In our testing, we considered "large" to be at least $\frac{5 \pi}{6}$ in which case that path was truncated from further computation. 


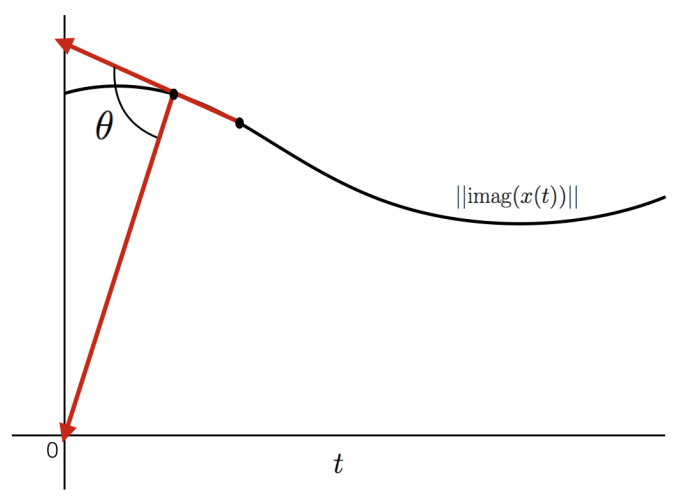

(a)

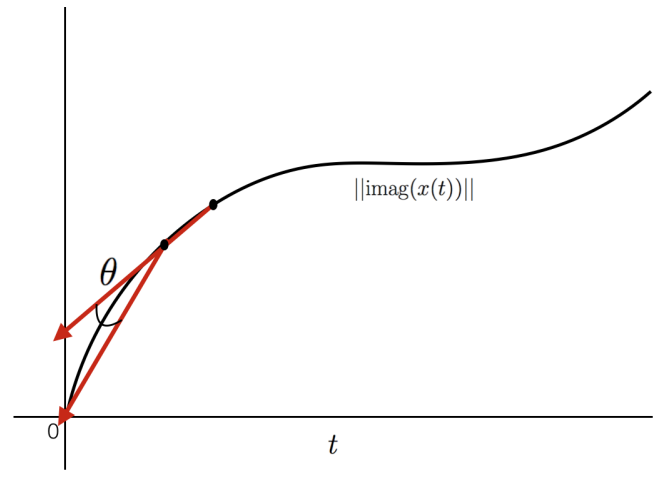

(b)

Figure 10: Schematic representation of (a) large angle leading toward a non-real solution and (b) small angle that leads to a real solution.

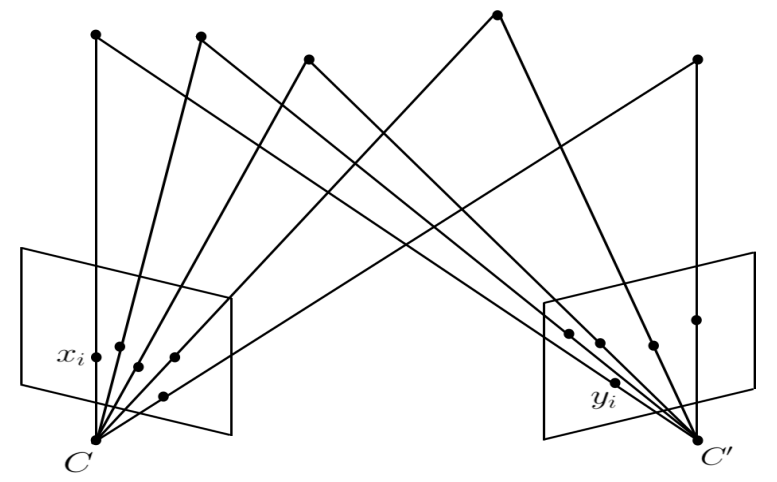

Figure 11: Schematic drawing of the 5-point image reconstruction problem.

\section{Applications in computer vision}

In order to demonstrate the three aspects of homotopy continuation investigated, namely adaptive affine coordinate patches, adaptively selected well-constrained subsystems, and truncation for paths heading to nonreal solutions, we consider two problems in computer vision. These problems are so-called minimal problems in computer vision in that they generically have finitely many solutions with the current techniques unstable for small problem sizes as well as require many assumptions and simplifications [17, 18]. We describe two minimal problems in Sections 7.1 and 7.2, with Section 7.3 presenting the computational results.

\subsection{5-point problem}

The 5-point problem involves two cameras where both images have 5 corresponding points mapping from a 3D object in space $8,16,24,29$. With the 5 corresponding point pairs $x_{i}$ for camera $C$ and $y_{i}$ for camera $C^{\prime}$ as shown in Figure 11, this problem computes the relative position and orientation 

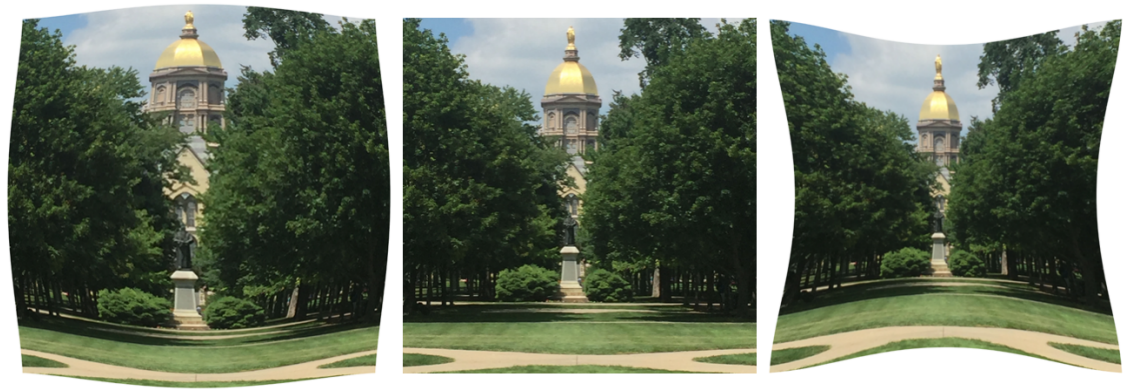

Figure 12: Image reconstruction with radial distortion.

of two calibrated cameras using the polynomial system:

$$
\left[\begin{array}{cc}
2 E E^{T} E-\operatorname{trace}\left(E E^{T}\right) E & \\
y_{i}^{T} E x_{i} & i=1, \ldots, 5
\end{array}\right] .
$$

As written, this system consists of 14 polynomials defined on $\mathbb{P}^{8}$ so that it is an overdetermined parameterized system defined on projective space which generically has 10 solutions.

\subsection{6-point problem}

The 6-point problem involves two cameras where both images have 6 corresponding points mapping from a 3D object in space with a radial distortion parameter $\lambda[5,18,30]$. Figure 12 shows images which have radial distortion. With the 6 corresponding point pairs $x_{i}$ for camera $C$ and $y_{i}$ for camera $C^{\prime}$, this problem computes the relative position and orientation of two calibrated cameras using the polynomial system:

$$
\left[\begin{array}{cc}
2 E E^{T} E-\operatorname{trace}\left(E E^{T}\right) E & \\
p_{i}(\lambda)^{T} E q_{i}(\lambda) & i=1, \ldots, 6
\end{array}\right]
$$

where

$$
p_{i}(\lambda)=\left[\begin{array}{c}
y_{i} \\
1+\lambda\left\|y_{i}\right\|_{2}^{2}
\end{array}\right] \quad \text { and } \quad q_{i}(\lambda)=\left[\begin{array}{c}
x_{i} \\
1+\lambda\left\|x_{i}\right\|_{2}^{2}
\end{array}\right] .
$$

As written, this system consists of 15 polynomials defined on $\mathbb{P}^{8} \times \mathbb{C}$ so that it is an overdetermined parameterized system defined on a product of a projective space and an affine space which generically has 52 solutions.

\subsection{Results}

We implemented Algorithm 1 to solve the 5-point and 6-point problems 1 using homotopy continuation with the data resulting from solving 100 random instances are summarized Tables 3 and 4 . respectively. In Algorithm 1, the predictor-corrector step we utilized was the classical $4^{\text {th }}$ order Runge-Kutta predictor with a maximum of 3 Newton iterations. These tables summarize average number of steps per path, average number of arithmetic operations per parameter instance to be solved, and average time (in seconds) per instance for various patching (Section 3) and randomization (Section 4) strategies together with path truncation (Section 6).

\footnotetext{
${ }^{1}$ Available at http://dx.doi.org/10.7274/ROC53HXK
} 


\begin{tabular}{l|c|c|c|c|c|c} 
& FP/FR & OP/FR & CWP/FR & OP/PIR & CWP/LSR & CWP/LSR/ET \\
\hline \hline Avg. \# steps/path & 14.127 & 11.261 & 10.026 & 10.859 & 9.224 & 8.338 \\
Avg. \# operations & 53,646 & 53,886 & 34,733 & 41,038 & 30,827 & 44,908 \\
Avg. time (sec) & 0.3641 & 0.4449 & 0.3611 & 0.4637 & 0.2862 & 0.2608
\end{tabular}

Table 3: Summary of solving 100 random instances of the 5-point problem

\begin{tabular}{l|c|c|c|c|c|c} 
& FP/FR & OP/FR & CWP/FR & OP/PIR & CWP/LSR & CWP/LSR/ET \\
\hline \hline Avg. \# steps/path & 29.667 & 23.361 & 24.717 & 20.224 & 21.721 & 15.746 \\
Avg. \# operations & $11,649,000$ & $9,029,600$ & $7,289,400$ & $7,740,500$ & $6,340,300$ & $4,700,900$ \\
Avg. time (sec) & 5.810 & 4.526 & 5.302 & 4.259 & 4.832 & 3.707
\end{tabular}

Table 4: Summary of solving 100 random instances of the 6-point problem

$\mathrm{FP}=$ fixed random patch (Section 3.1), OP = adaptive orthogonal patch (Section 3.2), $\mathrm{CWP}=$ adaptive coordinate-wise patch (Section 3.3), FR = fixed randomization (Section 4.1), $\mathrm{PIR}=$ adaptive pseudoinverse randomization (Section 4.2), LSR $=$ adaptive leverage score randomization (Section 4.3), and ET = early truncation (Section 6).

The results show that the combination of adaptive coordinate-wise patching, leverage score randomization, and early truncation had both the lowest average number of steps per path and average solving time for both the 5-point and 6-point problems. In particular, for the 5-point problem, this combination yielded about a $40 \%$ decrease in average number of steps per path over tracking using a fixed random patch with fixed randomization. For the 6-point problem, the decrease was $47 \%$ due to the larger size and more paths which could be truncated. In particular, every adaptive method results in reducing the average number of steps per path compared with using a fixed random patch with fixed randomization.

\section{Conclusion}

Three aspects of using homotopy continuation to solve parameterized systems were investigated: selecting affine coordinate patches, selecting well-constrained subsystems, and truncating paths which appear to be ending at nonreal solutions. The results in Section 7 demonstrate substantial improvement by using adaptive selections over fixed random choices in path tracking.

\section{Acknowledgments}

The authors would like to thank Sameer Agarwal for his expertise in minimal problems, their implementation, and suggestions regarding the use of numerical algebraic geometry. We would also like to thank Alan Liddell, Ilse Ipsen, and Tim Kelley for helpful discussions.

\section{References}

[1] A.N. Al-Khateeb, J.M. Powers, S. Paolucci, A.J. Sommese, J.A. Diller, J.D. Hauenstein, and J. Mengers, One-dimensional slow invariant manifolds for spatially homogeneous reactive systems. J. Chem. Phys., 131, 2009. 
[2] D.J. Bates, D.A. Brake, and M.E. Niemerg, Paramotopy: Parameter homotopies in parallel. Available at paramotopy.com.

[3] D.J. Bates, J.D. Hauenstein, A.J. Sommese, and C.W. Wampler, Bertini: Software for numerical algebraic geometry. Available at bertini.nd.edu.

[4] D.J. Bates, J.D. Hauenstein, A.J. Sommese, and C.W. Wampler, Numerically Solving Polynomial Systems with Bertini. SIAM, 2013.

[5] M. Byröd, K. Josephson, and K. Åström, Improving numerical accuracy of Gröbner basis polynomial equation solvers. In ICCV07, IEEE, 2007, pp. 1-8.

[6] M. Demazure, Sur deux problemes de reconstruction. INRIA, 1988.

[7] P. Deuflhard, Newton Methods for Nonlinear Problems: Affine Invariance and Adaptive Algorithms. Springer Series in Computational Mathematics, 35, 2004.

[8] O.D. Faugeras and S.J. Maybank, Motion from point matches: multiplicity of solutions. International Journal of Computer Vision, 4(3):225-246, 1990.

[9] M.A. Fischler and R.C. Bolles, Random sample consensus: a paradigm for model fitting with applications to image analysis and automated cartography. Commun. ACM, 24(6):381-395, 1981.

[10] J.D. Hauenstein and F. Sottile, Algorithm 921: alphaCertified: Certifying solutions to polynomial systems. ACM Trans. Math. Softw., 38(4):28, 2012.

[11] J.D. Hauenstein and C.W. Wampler, Isosingular sets and deflation. Found. Comput. Math., 13(3):371-403, 2013.

[12] J.D. Hauenstein, C.W. Wampler, and M. Pfurner, Synthesis of three-revolute spatial chains for body guidance. Mechanism and Machine Theory, 110:61-72, 2017.

[13] D.C. Hoaglin and R.E. Welsch, The hat matrix in regression and ANOVA. The American Statistician, 32(1):17-22, 1978.

[14] I.C.F. Ipsen and T. Wentworth, The effect of coherence on sampling from matrices with orthonormal columns, and preconditioned least squares problems. SIAM J. Matrix Anal. Appl., 35(4):1490-1520, 2014.

[15] A. Irschara, C. Zach, J. M. Frahm, and H. Bischof, From structure-from-motion point clouds to fast location recognition. CVPR, 2599-2606, 2009.

[16] E. Kruppa, Zur Ermittlung eines Objektes aus zwei Perspektiven mit innerer Orientierung. Sitzungsberichte der Mathematisch Naturwissenschaftlichen Kaiserlichen Akademie der Wissenschaften, 122:1939-1948, 1913.

[17] Z. Kúkelová, Algebraic Methods in Computer Vision. Czech Technical University, 2013.

[18] Z. Kúkelová and T. Pajdla, Two minimal problems for cameras with radial distortion. In ICCV07, IEEE, 2007, pp. 1-8. 
[19] B. Leibe, N. Cornelis, K. Cornelis, and L. Van Gool, Dynamic 3D scene analysis from a moving vehicle. In CVPR, IEEE, 2007, pp. 1-8.

[20] A. Leykin, J. Verschelde, and A. Zhao, Newton's method with deflation for isolated singularities of polynomial systems. Theoret. Comput. Sci. 359(1-3):111-122, 2006.

[21] A.P. Morgan, A transformation to avoid solutions at infinity for polynomial systems. Appl. Math. Comput., 18(1):77-86, 1986.

[22] A.P. Morgan, Solving Polynomial Systems Using Continuation for Engineering and Scientific Problems. Prentice-Hall, 1987.

[23] A.P. Morgan and A.J. Sommese, Coefficient-parameter polynomial continuation. Appl. Math. Comput., 29(2):12-160, 1989.

[24] D. Nistér, An efficient solution to the five-point relative pose problem. IEEE Transactions on Pattern Analysis and Machine Intelligence, 26(6):756-777, 2004.

[25] M. Shub and S. Smale, Complexity of Bézout's theorem. I: Geometric aspects. J. Amer. Math. Soc., 6(2):459-501, 1993.

[26] N. Snavely, S. M. Seitz, and R. Szeliski, Photo tourism: exploring photo collections in 3D. ACM SIGGRAPH, 835-846, 2006.

[27] A.J. Sommese and C.W. Wampler, Numerical algebraic geometry. Lectures in Appl. Math., 32:749-763, 1996.

[28] A.J. Sommese and C.W. Wampler, The Numerical Solution of Systems of Polynomials Arising in Engineering and Science. World Scientific, 2005.

[29] H. Stewénius, C. Engels, and D. Nistér, Recent developments on direct relative orientation. ISPRS Journal of Photogrammetry and Remote Sensing, 60(4):284-294, 2006.

[30] H. Stewénius, D. Nistér, F. Kahl, and F. Schaffalitzky, A minimal solution for relative pose with unknown focal length. Image and Vision Computing, 26(7):871-877, 2008.

[31] C.W. Wampler and A.J. Sommese, Numerical algebraic geometry and algebraic kinematics. Acta Numerica, 20:469-567, 2011. 